\title{
FUNCTIONAL RELATIONSHIPS BETWEEN PRICE AND VOLATILITY JUMPS AND THEIR CONSEQUENCES FOR DISCRETELY OBSERVED DATA
}

\author{
JEAN JACOD, ${ }^{*}$ Université Pierre et Marie Curie \\ CLAUDIA KLÜPPELBERG ${ }^{* * * * *}$ AND \\ GERNOT MÜLLER, ****** Technische Universität München
}

\begin{abstract}
Many prominent continuous-time stochastic volatility models exhibit certain functional relationships between price jumps and volatility jumps. We show that stochastic volatility models like the Ornstein-Uhlenbeck and other continuous-time CARMA models as well as continuous-time GARCH and EGARCH models all exhibit such functional relations. We investigate the asymptotic behaviour of certain functionals of price and volatility processes for discrete observations of the price process on a grid, which are relevant for estimation and testing problems.
\end{abstract}

Keywords: Barndorff-Nielsen Shephard model; CARMA; continuous-time GARCH; COGARCH; common jumps; high-frequency data; Itô semimartingale; Lévy process; Ornstein-Uhlenbeck process; stochastic volatility

2010 Mathematics Subject Classification: Primary 60G48; 60H30; 91 G70

Secondary 62G10; 62M02

\section{Introduction}

Models for financial data involving a stochastic volatility and allowing for sample path discontinuities in the volatility as well as in the underlying asset price (stock, index, exchange rate) have become more and more popular in recent years. Since Merton's paper [24] there has been an increasing number of publications showing empirical evidence of jumps in the asset prices: from the distribution of log-returns viewpoint, as in [1], [9], and [10], or from a nonparametric statistical viewpoint, as in [4], [6], [14], and [23] using multipower variations, or with other methods, as in [3] and [22]; see also the references in these papers.

Naturally, if the asset price process has jumps, one might suppose that the volatility process also exhibits jumps, which is supported by empirical evidence; cf. [17]. Starting from the assumption of jumps present in the price process as well as in the volatility process, it is important to investigate in a next step a possible relation between price jumps and volatility jumps. In a first statistical analysis [18] we have investigated whether, for such common jumps, some functions of the jump sizes are correlated.

Received 24 August 2012.

* Postal address: Institut de Mathématiques de Jussieu, Université Pierre et Marie Curie, 4 Place Jussieu, 75005 Paris, France. Email address: jean.jacod@upmc.fr

** Postal address: Centre for Mathematical Sciences, Technische Universität München, 85748 Garching, Germany.

*** Email address: cklu@ma.tum.de

*****Email address: mueller@ma.tum.de 
The present paper includes work presented by CK at the 2011 Applied Probability Trust lecture in Sheffield. The lecture comprised an overview of probabilistic results and statistical methods for the continuous-time GARCH (COGARCH) model, which was proposed in [19], and reviewed in [11], [20], and [21]. Instead of publishing another review paper here-after discussion with the Editor-we present rather new insights on stochastic volatility models with jumps, including the COGARCH model.

Many specific models which feature jumps in the price and volatility exhibit much more than correlated price and volatility jumps, they impose a functional relationship between these jumps, when they occur at the same time. For example, the following one-factor continuoustime models, sometimes including a leverage term, exhibit such functional relationships:

- the Ornstein-Uhlenbeck or BNS (Barndorff-Nielsen Shephard) model [5];

- the CARMA models [7], [25];

- the COGARCH model [13], [19]; and

- the ECOGARCH model [12].

All such models fit into a semimartingale framework, and the aim of this paper is to investigate the possibility of testing whether, in this general framework, a functional relationship occurs between the jumps in the price and volatility.

One problem which immediately arises is the structure of the data: the price is observed at discrete times only, typically at times $i \Delta_{n}$ for all integers $i$, up to the final horizon $T$, where $\Delta_{n}$ is a time lag, which is small. However, the price jumps are not really observed, although 'big' jumps are reasonably well known. For the volatility, the problem is much worse, because it is never observed per se: we need a preliminary estimation, which unfortunately is not really accurate in view of the typically available data. So, price jumps can only be detected when they are big enough, and even more so for volatility jumps. To tackle the problem of local volatility estimation, various methods have been proposed in different settings; see [2], [17], and the references therein.

On top of these 'discretization' errors, we have another problem: checking for a given functional relationship can be done as soon as there are at least two (joint) jumps, and this usually occurs in relatively short periods of time, if there are jumps at all.

Below, we propose a method for testing a (relatively) arbitrary functional relationship between price and volatility jumps, for those which occur simultaneously. As mentioned above, it has been shown in [17] that such common jumps are likely to happen. We then apply this method to the four different stochastic volatility models above. These models allow for joint upwards and (perhaps also) downwards jumps in the prices and the volatilities. This is done in the natural discrete observation scheme mentioned above, in spite of the estimation problems already mentioned.

The paper is organized as follows. In Section 2 we set the stage by formulating the (log) price and stochastic volatility models in terms of the full generality of a bivariate Itô semimartingale. We also formulate the functional relationships we want to investigate in detail in this paper. We introduce the discretized version of the process and recall the spot volatility estimator. Then we present the relevant statistics and prove a central limit theorem. In Section 3 we present the four model classes and their functional relationships between jump sizes in the price and volatility. We also explain the forms of the corresponding test statistics and the rejection mechanism. Finally, the test itself is presented in Section 4. 


\section{The model framework}

Our (nonparametric) stochastic volatility model is a 'joint' model for the (log) price $X$ and volatility $\sigma$. All processes are one-dimensional here and we set $c_{t}=\sigma_{t}^{2}$; the model is really for the pair $(X, c)$. We have a filtered probability space $\left(\Omega, \mathcal{F},\left(\mathcal{F}_{t}\right)_{t \geq 0}, \mathrm{P}\right)$, on which

$$
\begin{aligned}
X_{t}= & X_{0}+\int_{0}^{t} b_{s} \mathrm{~d} s+\int_{0}^{t} \sigma_{s} \mathrm{~d} W_{s}+\int_{0}^{t} \int_{E}\left(\delta(s, z) \mathbf{1}_{\{|\delta(t, z)| \leq 1\}}\right)(\mu-v)(\mathrm{d} s, \mathrm{~d} z) \\
& +\int_{0}^{t} \int_{E}\left(\delta(s, z) \mathbf{1}_{\{|\delta(t, z)|>1\}}\right) \mu(\mathrm{d} s, \mathrm{~d} z), \\
c_{t}= & c_{0}+\int_{0}^{t} \tilde{b}_{s} \mathrm{~d} s+\int_{0}^{t} \tilde{\sigma}_{s} \mathrm{~d} W_{s}+\int_{0}^{t} \tilde{\sigma}_{s}^{\prime} \mathrm{d} W_{s}^{\prime}+\int_{0}^{t} \int_{E}\left(\tilde{\delta}(s, z) \mathbf{1}_{\{|\tilde{\delta}(t, z)| \leq 1\}}\right)(\mu-v)(\mathrm{d} s, \mathrm{~d} z) \\
& +\int_{0}^{t} \int_{E}\left(\tilde{\delta}(s, z) \mathbf{1}_{\{|\tilde{\delta}(t, z)|>1\}}\right) \mu(\mathrm{d} s, \mathrm{~d} z) .
\end{aligned}
$$

Here, $W$ and $W^{\prime}$ are two independent standard Brownian motions, $\mu=\mu(\omega, \mathrm{d} t, \mathrm{~d} z)$ is a Poisson random measure on $\mathbb{R}_{+} \times E$, where $(E, \mathcal{E})$ is an auxiliary Polish space, and the intensity measure of $\mu$ is $v(\mathrm{~d} t, \mathrm{~d} x)=\mathrm{d} t \otimes \lambda(\mathrm{d} z)$ for some $\sigma$-finite measure $\lambda$ on $(E, \mathcal{E})$ (there is a lot of freedom for choosing $\mu, E$, and $\lambda$ ). The 'coefficients' of the model are the two drifts $b_{t}$ and $\tilde{b}_{t}$, the two processes $\tilde{\sigma}_{t}$ and $\tilde{\sigma}_{t}^{\prime}$, and the two functions $\delta$ and $\tilde{\delta}$ on $\Omega \times \mathbb{R}_{+} \times E$.

Equivalently, the pair $(X, c)$ is an Itô semimartingale, whose characteristics $(B, C, v)$ have the form

$$
\begin{gathered}
B_{t}^{1}=\int_{0}^{t} b_{s} \mathrm{~d} s, \quad B_{t}^{2}=\int_{0}^{t} \tilde{b}_{s} \mathrm{~d} s, \\
C_{t}^{11}=\int_{0}^{t} c_{s} \mathrm{~d} s, \quad C_{t}^{12}=C_{t}^{21}=\int_{0}^{t} \sigma_{s} \tilde{\sigma}_{s} \mathrm{~d} s, \quad C_{t}^{22}=\int_{0}^{t}\left(\tilde{\sigma}_{s}^{2}+\tilde{\sigma}_{s}^{\prime 2}\right) \mathrm{d} s, \\
v(\omega ; \mathrm{d} t, \mathrm{~d} x, \mathrm{~d} y)=\mathrm{d} t F_{t}(\omega ; \mathrm{d} x, \mathrm{~d} y),
\end{gathered}
$$

where $F_{t}(\omega ; \cdot)$ is the image of $\lambda$ by $z \mapsto(\delta(\omega, t, z), \tilde{\delta}(\omega, t, z))$, restricted to $\mathbb{R}^{2} \backslash\{0\}$.

This formulation is general enough to accommodate a large variety of situations.

- Cases without or with leverage for the 'continuous part', according to whether $\tilde{\sigma}_{t}$ is identically 0 or not.

- The case where $X$ and $\sigma$ do not jump together, if $F_{t}$ is supported by the two axes $\mathbb{R} \times\{0\}$ and $\{0\} \times \mathbb{R}$.

- The case where $X$ and $\sigma$ have common jumps, when $F_{t}$ puts some positive mass outside of the two axes, including the case when they always jump together, when $F_{t}$ does not charge the two axes.

\subsection{Functional relationships}

The bivariate semimartingale $(X, c)$ has characteristics $(2.1)$, where the processes $b_{t}, \tilde{b}_{t}, \tilde{\sigma}_{t}$, and $\tilde{\sigma}_{t}^{\prime}$ should be progressively measurable, and the functions $\delta$ and $\tilde{\delta}$ should be predictable. All these terms are 'not too large', so all the integrals make sense, and they should also be such that $c_{t} \geq 0$ identically. We refer the reader to [16] for all unexplained, but classical, notions or notation.

In particular, we may have a functional relationship between the jumps of $X$ and $c$, at least when the jumps of $X$ belong to some subset $A$ of $\mathbb{R} \backslash\{0\}$ (with possibly $A=\mathbb{R} \backslash\{0\}$ ); this can 
be expressed as

$$
\phi\left(c_{t-}, c_{t}\right)=\gamma \psi\left(X_{t-}, X_{t}\right) \quad \text { if } \Delta X_{t} \in A
$$

for some given (known) functions $\psi$ and $\phi$ and a (usually unknown) parameter $\gamma \in \mathbb{R}$. An equivalent formulation of this property is that the restriction of $F_{t}$ to the set $A \times \mathbb{R}$ is supported by the 'curve' $\left\{(x, y): \phi\left(c_{t-}, c_{t-}+y\right)=\gamma \psi\left(X_{t-}, X_{t-}+x\right)\right\}$.

The specific assumption which we need is as follows, where $r$ is a given number inside $[0,2)$.

Assumption (A-r). (a) The processes $b_{t}, \tilde{b}_{t}, \tilde{\sigma}_{t}$, and $\tilde{\sigma}_{t}^{\prime}$ are locally bounded.

(b) There exist a locally bounded process $\Gamma_{t}$ and a (nonrandom) function $\chi \geq 0$ such that $|\delta(\omega, t, z)| \leq \Gamma_{t}(\omega) \chi(z),|\tilde{\delta}(\omega, t, z)| \leq \Gamma_{t}(\omega) \chi(z)$, and $\int_{E}\left(\chi(z)^{r} \wedge 1\right) \lambda(\mathrm{d} z)<\infty$.

(c) The processes $c_{t}$ and $c_{t-}$ do not vanish.

The (finite) time horizon $T$ here is fixed. We want to investigate (2.2), restricted to the time interval $[0, T]$, and its consequences for a realistic modelling. In the examples of Section 3 , but also in most cases one can think of, relation (2.2) should hold for all jumps of $X$, or for all positive jumps, or for all negative jumps, thus taking leverage between price and volatility jumps into account. Since very small jumps of $X$ cannot be realistically inferred, these considerations lead us to take for $A$ one of the following three sets:

$$
A=(-\infty,-\varepsilon), \quad A=(\varepsilon, \infty), \quad \text { or } \quad A=(-\infty,-\varepsilon) \cup(\varepsilon, \infty)
$$

for some given $\varepsilon>0$. We make the following additional assumption:

$X$ has almost surely (a.s.) no jump with size $\Delta X_{t}$ in the boundary $\partial A$ of $A$.

This is quite weak: typically, the first marginal $F_{t}(\mathrm{~d} x, \mathbb{R})$ of the Lévy measures has a density, implying that (2.4) is satisfied when $A$ has the form (2.3), and otherwise it is satisfied for all $\varepsilon>0$ except countably many values.

As for the functions $\psi$ and $\phi$ occurring in (2.2), and although weaker assumptions are possible for parts of what follows, we will assume throughout the following (with obvious notation for the derivatives or partial derivatives) that

$\psi$ and $\phi$ are $C^{2}$ functions, with $\psi(x, y)>0$ if $x \neq y$,

$$
\text { and }\left|\phi_{1}^{\prime}(x, y)\right|+\left|\phi_{2}^{\prime}(x, y)\right|>0 \text { for all } x, y \in \mathbb{R} \text {. }
$$

Obviously, (2.2) is meaningless if no jump of $X$ before time $T$ has size in $A$, and it is automatically satisfied if there is a single such jump, say at time $S$, because it then holds with $\gamma=\phi\left(c_{S-}, c_{S}\right) / \psi\left(X_{S-}, X_{S}\right)$. Therefore, we define

$$
\Omega_{T}^{A}=\left\{\omega \in \Omega: \sum_{s \leq T} \mathbf{1}_{\left\{\Delta X_{s}(\omega) \in A\right\}} \geq 2\right\}
$$

as the set on which there are at least two jumps of $X$ within $[0, T]$ with size in $A$. Furthermore, define

$$
\begin{gathered}
\Omega_{T}^{(A, \gamma)}=\left\{\omega \in \Omega_{T}^{A}: \text { there is some } \gamma(\omega) \in \mathbb{R} \text { such that, if } s \in(0, T] \text { and } \Delta X_{s}(\omega) \in A,\right. \\
\text { then } \left.\phi\left(\sigma_{s-}(\omega), \sigma_{s}(\omega)\right)=\gamma(\omega) \psi\left(X_{s-}(\omega), X_{s}(\omega)\right)\right\} .
\end{gathered}
$$

Being in $\Omega_{T}^{(A, \gamma)}$ seems more general than having (2.2), because it allows for $\gamma=\gamma(\omega)$ to be random; however, in practice this is immaterial because, upon observing a single path of $(X, \sigma)$, it is of course impossible to decide whether $\gamma$ is random or not. 


\subsection{The discretized process}

For any process $Y$, we write

$$
\Delta_{i}^{n} Y=Y_{i \Delta_{n}}-Y_{(i-1) \Delta_{n}}
$$

we refer the reader to [15] for background reading. We choose two numbers $\varpi, \rho \in\left(0, \frac{1}{2}\right)$ and two sequences $u_{n}>0$ and $k_{n} \geq 1$ such that, for some constant $C>1$,

$$
\frac{\Delta_{n}^{\varpi}}{C} \leq u_{n} \leq C \Delta_{n}^{\varpi}, \quad \frac{1}{C \Delta_{n}^{\rho}} \leq k_{n} \leq \frac{C}{\Delta_{n}^{\rho}} .
$$

The following variables serve as 'local estimators' of the squared volatility $c_{t}=\sigma_{t}^{2}$ for a window of size $k_{n} \Delta_{n}$ starting at time $i \Delta_{n}$ (cf. [15, Equation (9.3.1)]):

$$
\hat{c}_{i}^{n}=\frac{1}{k_{n} \Delta_{n}} \sum_{j=1}^{k_{n}}\left|\Delta_{i+j}^{n} X\right|^{2} \mathbf{1}_{\left\{\left|\Delta_{i+j}^{n} X\right| \leq u_{n}\right\}} .
$$

It is always nonnegative, and even a.s. positive under Assumption (A-r)(a), which implies that $\Delta_{i}^{n} X \neq 0$ a.s. for all $i$ and $n$.

The functions $\phi$ and $\psi$ satisfying (2.5) and $A$ satisfying (2.3) are given, and we assume that (2.4) holds. For any two integers $p, q \geq 0$, we set

$$
\begin{gathered}
U(p, q)_{T}=\sum_{s \leq T} \mathbf{1}_{A}\left(\Delta X_{S}\right) \psi\left(X_{s-}, X_{S}\right)^{p} \phi\left(c_{s-}, c_{s}\right)^{q}, \\
\Phi_{T}=U(1,1)_{T}^{2}-U(2,0)_{T} U(0,2)_{T} .
\end{gathered}
$$

Being in $\Omega_{T}^{(A, \gamma)}$ amounts to being in $\Omega_{T}^{A}$, plus having $U(0,2)_{T}-2 \gamma U(1,1)_{T}+\gamma^{2} U(2,0)_{T}=0$ for some $\gamma=\gamma(\omega)$. Since $\gamma \mapsto U(0,2)_{T}-2 \gamma U(1,1)_{T}+\gamma^{2} U(2,0)_{T}$ is quadratic and always nonnegative, we thus have

$$
\Omega_{T}^{(A, \gamma)}=\Omega_{T}^{A} \cap\left\{\Phi_{T}=0\right\} .
$$

This leads us to construct a sequence of statistics which converges to $\Phi_{T}$. For this, we set

$$
U^{n}(p, q)_{T}=\sum_{i=k_{n}+1}^{\left[T / \Delta_{n}\right]-k_{n}} \mathbf{1}_{A}\left(\Delta_{i}^{n} X\right) \psi\left(X_{(i-1) \Delta_{n}}, X_{i \Delta_{n}}\right)^{p} \phi\left(\hat{c}_{i-k_{n}-1}^{n}, \hat{c}_{i}^{n}\right)^{q}
$$

and

$$
\Phi_{T}^{n}=U^{n}(1,1)_{T}^{2}-U^{n}(2,0)_{T} U^{n}(0,2)_{T} .
$$

Observe that, by the Cauchy-Schwarz inequality, we necessarily have $\Phi_{T} \leq 0$ and $\Phi_{T}^{n} \leq 0$.

\subsection{Some limit theorems}

We give the asymptotic behaviour of $\Phi_{T}^{n}$. The properties in (2.5) are in force below, without special mention. We have consistency (that is, $\Phi_{T}^{n} \stackrel{\mathrm{P}}{\rightarrow} \Phi_{T}$ - see Theorem 2.1 below), but also present a central limit theorem, and this necessitates some preparation.

We consider an auxiliary probability space $\left(\Omega^{\prime}, \mathcal{F}^{\prime}, \mathrm{P}^{\prime}\right)$, on which we have a double sequence $\left(V_{m}^{-}, V_{m}^{+}\right)_{m \geq 1}$ of independent and identically distributed (i.i.d.) $\mathcal{N}(0,1)$ variables. We denote 
by $S_{1}, S_{2}, \ldots$ the successive times at which a jump of $X$ with size within $A$ occurs, so this sequence increases to $\infty$. Then we define an extension $(\tilde{\Omega}, \tilde{\mathcal{F}}, \tilde{\mathrm{P}})$ of $(\Omega, \mathcal{F}, \mathrm{P})$ by setting

$$
\tilde{\Omega}=\Omega \times \Omega^{\prime}, \quad \tilde{\mathcal{F}}=\mathcal{F} \otimes \mathcal{F}^{\prime}, \quad \tilde{\mathrm{P}}=\mathrm{P} \otimes \mathrm{P}^{\prime} .
$$

Any variable or process defined on $\Omega$ or $\Omega^{\prime}$ is extended to $\tilde{\Omega}$ in the usual way, without a change of notation.

We then recall the notion of stable convergence in law. This kind of convergence is slightly stronger than convergence in law. For details and extensions, see [15, Section 2.2.1]. A formal definition is as follows. Let $\left(V_{n}\right)_{n \geq 1}$ be random variables on $(\Omega, \mathcal{F}, \mathrm{P})$, and let $V$ be a variable defined on the extension $(\tilde{\Omega}, \tilde{\mathcal{F}}, \tilde{\mathrm{P}})$, as defined in (2.12). Then $V_{n}$ converges to $V$ stably in law $\left(V_{n} \stackrel{\mathcal{L}-(s)}{\longrightarrow} V\right)$ if $\mathrm{E}\left[Y f\left(V_{n}\right)\right] \rightarrow \tilde{\mathrm{E}}[Y f(V)]$ as $n \rightarrow \infty$ for all bounded $\mathcal{F}$-measurable random variables $Y$ and all bounded Lipschitz functions $f$. In contrast with the usual convergence in law, saying that $V_{n}$ converges stably in law to $V$ 'in restriction to a set $B$ ' is meaningful, and means that $\mathrm{E}\left[Y f\left(V_{n}\right)\right] \rightarrow \tilde{\mathrm{E}}[Y f(V)]$ for all $Y$ and $f$ as above, with, in addition, $Y=0$ outside $B$.

We have $(\psi, \phi)$ satisfying (2.5), and $A$ of the form (2.3) with $\varepsilon>0$ satisfying (2.4). We denote by $\left(T_{m}\right)_{m \geq 1}$ the successive jump times of the counting process $N_{t}^{\prime}=\mu((0, t] \times$ $\{z: \chi(z)>\varepsilon / 2\})$ (a Poisson process with parameter $\lambda(\{z: \chi(z)>\varepsilon / 2\})$-recall that the function $\chi$ is given in Assumption $(\mathrm{A}-r)(\mathrm{b}))$. We introduce the following notation:

$$
\begin{array}{ll}
i(n, m) \text { is the unique (random) integer with }(i(n, m)-1) \Delta_{n}<T_{m} \leq i(n, m) \Delta_{n}, \\
\hat{c}^{n}(m-)=\hat{c}_{i(n, m)-k_{n}-1}^{n}, & \hat{c}^{n}(m+)=\hat{c}_{i(n, m)}^{n}, \\
\kappa_{m-}^{n}=\sqrt{k_{n}}\left(\hat{c}^{n}(m-)-c_{T_{m}-}\right), & \kappa_{m+}^{n}=\sqrt{k_{n}}\left(\hat{c}^{n}(m+)-c_{T_{m}}\right), \\
\kappa_{m-}^{\prime n}=\frac{1}{\sqrt{\Delta_{n}}}\left(X_{T_{m}-}-X_{(i(n, m)-1) \Delta_{n}}\right), & \kappa_{m+}^{\prime n}=\frac{1}{\sqrt{\Delta_{n}}}\left(X_{i(n, m) \Delta_{n}}-X_{T_{m}}\right), \\
\kappa_{m}^{\prime n}=\kappa_{m-}^{\prime n}+\kappa_{m+}^{\prime n} . &
\end{array}
$$

Then we first have

each sequence $\left(\kappa_{m \pm}^{\prime n}\right)_{m \geq 1}$ is bounded in probability.

Moreover, under Assumption (A-r) and if $\rho$ in (2.7) satisfies

$$
\rho \leq \frac{2-r}{r}
$$

then from Theorem 13.3.3 of [15] for $\beta=0$ we know that

$$
\left(\kappa_{m-}^{n}, \kappa_{m+}^{n}\right)_{m \geq 1} \stackrel{\mathcal{L}-(s)}{\longrightarrow}\left(c_{T_{m}-} \sqrt{2} V_{m}^{-}, c_{T_{m}} \sqrt{2} V_{m}^{+}\right)_{m \geq 1}
$$

We also set

$$
N_{t}=\sum_{m \geq 1} \mathbf{1}_{\left\{S_{m} \leq t\right\}}=\sum_{s \leq t} \mathbf{1}_{\left\{\Delta X_{s} \in A\right\}}
$$

for the number of jumps of $X$ with size in $A$, up to time $t$. Recalling that $A$ satisfies (2.3), by Assumption (A-r)(b) we have $N_{t} \leq N_{t}^{\prime}$, and in fact every $S_{m}$ equals some $T_{m^{\prime}}$ (with usually a random index $\left.m^{\prime}\right)$. 
Theorem 2.1. Suppose that Assumption (A-r) for some $r \in[0,2),(2.4)$, and (2.5) hold. Then, for $\Phi_{T}$ as in (2.8), we have, as $n \rightarrow \infty$,

$$
\Phi_{T}^{n} \stackrel{\mathrm{P}}{\rightarrow} \Phi_{T}
$$

Furthermore, if $\rho$ in (2.7) satisfies (2.14), we have the following stable convergence in law as $n \rightarrow \infty$ :

$$
k_{n} \Phi_{T}^{n} \stackrel{\mathcal{L}-(s)}{\longrightarrow} \bar{\Phi}_{T} \quad \text { in restriction to the set } \Omega_{T}^{(A, \gamma)} .
$$

Here

$$
\begin{aligned}
\bar{\Phi}_{T}= & 2\left(\sum_{m=1}^{N_{T}} \psi\left(X_{S_{m}-}, X_{S_{m}}\right)\left(c_{S_{m}-} \phi_{1}^{\prime}\left(c_{S_{m}-}, c_{S_{m}}\right) V_{m}^{-}+c_{S_{m}} \phi_{2}^{\prime}\left(c_{S_{m}-}, c_{S_{m}}\right) V_{m}^{+}\right)\right)^{2} \\
& -2\left(\sum_{m=1}^{N_{T}} \psi\left(X_{S_{m}-}, X_{S_{m}}\right)^{2}\right)\left(\sum_{m=1}^{N_{T}}\left(c_{S_{m}-} \phi_{1}^{\prime}\left(c_{S_{m}-}, c_{S_{m}}\right) V_{m}^{-}+c_{S_{m}} \phi_{2}^{\prime}\left(c_{S_{m}-}, c_{S_{m}}\right) V_{m}^{+}\right)^{2}\right) .
\end{aligned}
$$

Moreover, $\bar{\Phi}_{T}<0$ a.s. on $\Omega_{T}^{\varepsilon}$ and $\bar{\Phi}_{T}=0$ a.s. on the complement $\left(\Omega_{T}^{\varepsilon}\right)^{\mathrm{c}}$.

Proof. In view of (2.9) and (2.11), for $\Phi_{T}^{n} \stackrel{\text { P }}{\rightarrow} \Phi_{T}$, it is enough to prove that, for all integers $p, q \geq 0$, we have

$$
U^{n}(p, q)_{T} \stackrel{\mathrm{P}}{\rightarrow} U(p, q)_{T} .
$$

From Assumption (A-r) and the definition of the stopping times $T_{m}$, which imply that every $s$ with $\left|\Delta X_{S}\right|>\varepsilon / 2$ is equal to some (unique) $T_{m}$, we see that, for all $n \geq n_{T}(\omega)$ for some finite variable $n_{T}$, we have (we use the fact that $A$ is open and no jump of $X$ lies on its boundary $\partial A$ )

$$
\begin{gathered}
T_{1}>2 k_{n} \Delta_{n}, \quad T_{N_{T}^{\prime}}<T-2 k_{n} \Delta_{n}, \quad 1 \leq m \leq N_{T}^{\prime} \quad \Longrightarrow \quad T_{m}-T_{m-1}>3 k_{n} \Delta_{n}, \\
i \in\left\{1, \ldots,\left[T / \Delta_{n}\right]\right\} \backslash\left\{i(n, 1), \ldots, i\left(n, N_{T}^{\prime}\right)\right\} \quad \Longrightarrow \quad\left|\Delta_{i}^{n} X\right| \leq \varepsilon, \\
1 \leq m \leq N_{T}^{\prime} \Longrightarrow\left\{\begin{array}{l}
\Delta_{i(n, m)}^{n} X \in A \\
\Delta_{i(n, m)}^{n} X \notin A
\end{array} \text { if } \Delta X_{T_{m}} \in A\right.
\end{gathered}
$$

Hence, if $n \geq n_{T}$, all summands in (2.10) are 0 except those corresponding to $i=i(n, m)$,

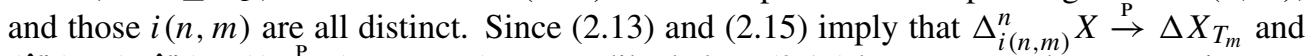
$\left(\hat{c}^{n}(m-), \hat{c}^{n}(m+)\right) \stackrel{\mathrm{P}}{\rightarrow}\left(c_{T_{m}-}, c_{T_{m}}\right)$, we readily deduce (2.17) because $\phi$ and $\psi$ are continuous.

Next, we introduce the simplifying notation

$$
v_{n}=\frac{1}{\sqrt{k_{n}}}, \quad \eta_{m}=\left(c_{T_{m}-}, c_{T_{m}}\right), \quad \eta_{m}^{\prime}=\left(X_{T_{m}-}, X_{T_{m}}\right) .
$$

Using (2.18) again, we see that

$$
n \geq n_{T} \quad \Longrightarrow \quad U^{n}(p, q)_{T}=\sum_{m=1}^{N_{T}^{\prime}} \zeta(p, q)_{m}^{n},
$$

where $\zeta(p, q)_{m}^{n}=\mathbf{1}_{A}\left(\Delta X_{T_{m}}\right) \psi\left(X_{T_{m}-}-\sqrt{\Delta_{n}} \kappa_{m-}^{\prime n}, X_{T_{m}}+\sqrt{\Delta_{n}} \kappa_{m+}^{\prime n}\right)^{p}$

$$
\times \phi\left(c_{T_{m}-}+v_{n} \kappa_{m-}^{n}, c_{T_{m}}+v_{n} \kappa_{m+}^{n}\right)^{q} .
$$


As before, we have

$$
\zeta(p, q)_{m}^{n} \stackrel{\mathrm{P}}{\rightarrow} \zeta(p, q)_{m}:=\mathbf{1}_{A}\left(\Delta X_{T_{m}}\right) \psi\left(\eta_{m}^{\prime}\right)^{p} \phi\left(\eta_{m}\right)^{q} .
$$

We use the notation $Y_{n}=\mathrm{o}_{P}\left(a_{n}\right)$ for a sequence $a_{n}$ of positive numbers if $Y_{n} / a_{n} \stackrel{\mathrm{P}}{\rightarrow} 0$. We recall once more that the variables $\sqrt{\Delta_{n}} \kappa_{m \pm}^{\prime n}$ and $v_{n} \kappa_{m \pm}^{n}$ tend to 0 in probability. Then, with obvious notation for the derivatives, and since $\sqrt{\Delta_{n}}=\mathrm{o}\left(v_{n}\right)$ by (2.7), we deduce from (2.19) that

$$
\begin{aligned}
\zeta(p, q)_{m}^{n}= & \zeta(p, q)_{m} \\
& +\mathbf{1}_{A}\left(\Delta X_{T_{m}}\right)\left[p \sqrt{\Delta_{n}} \psi\left(\eta_{m}^{\prime}\right)^{p-1} \phi\left(\eta_{m}\right)^{q}\left(\psi_{2}^{\prime}\left(\eta_{m}^{\prime}\right) \kappa_{m+}^{\prime n}-\psi_{1}^{\prime}\left(\eta_{m}^{\prime}\right) \kappa_{m-}^{\prime n}\right)\right. \\
& +q v_{n} \psi\left(\eta_{m}^{\prime}\right)^{p} \phi\left(\eta_{m}\right)^{q-1}\left(\phi_{1}^{\prime}\left(\eta_{m}\right) \kappa_{m-}^{n}+\phi_{2}^{\prime}\left(\eta_{m}\right) \kappa_{m+}^{n}\right) \\
& +\frac{q(q-1)}{2} v_{n}^{2} \psi\left(\eta_{m}^{\prime}\right)^{p} \phi\left(\eta_{m}\right)^{q-2}\left(\phi_{1}^{\prime}\left(\eta_{m}\right) \kappa_{m-}^{n}+\phi_{2}^{\prime}\left(\eta_{m}\right) \kappa_{m+}^{n}\right)^{2} \\
& +\frac{q}{2} v_{n}^{2} \psi\left(\eta_{m}^{\prime}\right)^{p} \phi\left(\eta_{m}\right)^{q-1} \\
& \left.\times\left(\phi_{11}^{\prime \prime}\left(\eta_{m}\right)\left(\kappa_{m-}^{n}\right)^{2}+2 \phi_{12}^{\prime \prime}\left(\eta_{m}\right) \kappa_{m-}^{n} \kappa_{m+}^{n}+\phi_{22}^{\prime \prime}\left(\eta_{m}\right)\left(\kappa_{m+}^{n}\right)^{2}\right)\right] \\
& +\mathrm{o} P\left(v_{n}^{2}\right) .
\end{aligned}
$$

At this stage we set

$$
Y_{n}^{j}(p, q)_{T}= \begin{cases}\sum_{m=1}^{N_{T}^{\prime}} \mathbf{1}_{A}\left(\Delta X_{T_{m}}\right) \psi\left(\eta_{m}^{\prime}\right)^{p} \phi\left(\eta_{m}\right)^{q}\left(\psi_{2}^{\prime}\left(\eta_{m}^{\prime}\right) \kappa_{m+}^{\prime n}-\psi_{1}^{\prime}\left(\eta_{m}^{\prime}\right) \kappa_{m-}^{\prime n}\right) & \text { if } j=1, \\ \sum_{m=1}^{N_{T}^{\prime}} \mathbf{1}_{A}\left(\Delta X_{T_{m}}\right) \psi\left(\eta_{m}^{\prime}\right)^{p} \phi\left(\eta_{m}\right)^{q}\left(\phi_{1}^{\prime}\left(\eta_{m}\right) \kappa_{m-}^{n}+\phi_{2}^{\prime}\left(\eta_{m}\right) \kappa_{m+}^{n}\right) & \text { if } j=2, \\ \frac{1}{2} \sum_{m=1}^{N_{T}^{\prime}} \mathbf{1}_{A}\left(\Delta X_{T_{m}}\right) \psi\left(\eta_{m}^{\prime}\right)^{p} \phi\left(\eta_{m}\right)^{q}\left(\phi_{1}^{\prime}\left(\eta_{m}\right) \kappa_{m-}^{n}+\phi_{2}^{\prime}\left(\eta_{m}\right) \kappa_{m+}^{n}\right)^{2} & \text { if } j=3, \\ \frac{1}{2} \sum_{m=1}^{N_{T}^{\prime}} \mathbf{1}_{A}\left(\Delta X_{T_{m}}\right) \psi\left(\eta_{m}^{\prime}\right)^{p} \phi\left(\eta_{m}\right)^{q} & \\ \times\left(\phi_{11}^{\prime \prime}\left(\eta_{m}\right)\left(\kappa_{m-}^{n}\right)^{2}+\phi_{22}^{\prime \prime}\left(\eta_{m}\right)\left(\kappa_{m+}^{n}\right)^{2}+2 \phi_{12}^{\prime \prime}\left(\eta_{m}\right) \kappa_{m-}^{n} \kappa_{m+}^{n}\right) & \text { if } j=4 .\end{cases}
$$

We use (2.19) to deduce that, for all $n \geq n_{0}$,

$$
\begin{aligned}
U^{n}(p, q)_{T}= & U(p, q)_{T}+p \sqrt{\Delta_{n}} Y_{n}^{1}(p-1, q)_{T}+v_{n} q Y_{n}^{2}(p, q-1)_{T} \\
& +v_{n}^{2} q\left((q-1) Y_{n}^{3}(p, q-2)_{T}+Y_{n}^{4}(p, q-1)\right)_{T}+\mathrm{o}_{P}\left(v_{n}^{2}\right) .
\end{aligned}
$$

Now we turn to proving the stable convergence in law of $\Phi_{T}^{n}$. For simplicity, we write $U=U(2,0)_{T}, U^{\prime}=U(0,2)_{T}$, and $U^{\prime \prime}=U(1,1)_{T}$. Observe that on the set $\Omega_{T}^{(A, \gamma)}$ there is a (possibly random) number $\gamma$ such that $U^{\prime}=\gamma U^{\prime \prime}=\gamma^{2} U$, and also $Y_{n}^{j}(p, q)_{T}=$ $\gamma^{q} Y_{n}^{j}(p+q, 0)_{T}$ for all $j=1,2,3,4$ and all $n$. Therefore, if $Y_{n}^{j}(p)_{T}=Y_{n}^{j}(p, 0)_{T}$, we deduce from (2.20) that

$$
\begin{aligned}
\Phi_{T}^{n}= & \left(U^{\prime \prime}\right)^{2}+2 U^{\prime \prime} \gamma \sqrt{\Delta_{n}} Y_{n}^{1}(1)_{T}+2 U^{\prime \prime} v_{n} Y_{n}^{2}(1)_{T}+v_{n}^{2}\left(Y_{n}^{2}(1)_{T}\right)^{2}+2 U^{\prime \prime} v_{n}^{2} Y_{n}^{4}(1)_{T} \\
& -U U^{\prime}-2 \sqrt{\Delta_{n}} U^{\prime} Y_{n}^{1}(2)_{T}-2 v_{n} \gamma U Y_{n}^{2}(1)-2 v_{n}^{2} U\left(Y_{n}^{3}(0)+\gamma Y_{n}^{4}(1)\right) \\
= & \Phi_{T}+v_{n}^{2}\left(\left(Y_{n}^{2}(1)\right)^{2}-2 U Y_{n}^{3}(0)\right)+\mathrm{o}_{P}\left(v_{n}^{2}\right) .
\end{aligned}
$$


Applying (2.15), we find that, in restriction to the set $\Omega_{T}^{(A, \gamma)}$, the variables $v_{n}^{-2} \Phi_{T}^{n}=k_{n} \Phi_{T}^{n}$ converge stably in law to

$$
\begin{aligned}
& 2\left(\sum_{m=1}^{N_{T}^{\prime}} \mathbf{1}_{A}\left(\Delta X_{T_{m}}\right) \psi\left(X_{T_{m}-}, X_{T_{m}}\right)\left(c_{T_{m}-} \phi_{1}^{\prime}\left(c_{T_{m}-}, c_{T_{m}}\right) V_{m}^{-}+c_{T_{m}} \phi_{2}^{\prime}\left(c_{T_{m}-}, c_{T_{m}}\right) V_{m}^{+}\right)\right)^{2} \\
& -2\left(\sum_{m=1}^{N_{T}^{\prime}} \mathbf{1}_{A}\left(\Delta X_{T_{m}}\right) \psi\left(X_{T_{m}-}, X_{T_{m}}\right)^{2}\right) \\
& \quad \times\left(\sum_{m=1}^{N_{T}^{\prime}} \mathbf{1}_{A}\left(\Delta X_{T_{m}}\right)\left(c_{T_{m}-} \phi_{1}^{\prime}\left(c_{T_{q} m-}, c_{T_{m}}\right) V_{m}^{-}+c_{T_{m}} \phi_{2}^{\prime}\left(c_{T_{m}-}, c_{T_{m}}\right) V_{m}^{+}\right)^{2}\right)
\end{aligned}
$$

Now, the variables $V_{m}^{ \pm}$are independent of $\mathcal{F}$, i.e. the $\mathcal{F}$-conditional distribution on the righthand side of (2.16) is the same as the $\mathcal{F}$-conditional distribution of (2.21). Therefore, $k_{n} \Phi_{T}^{n} \stackrel{\mathcal{L}-(s)}{\longrightarrow} \bar{\Phi}_{T}$ in restriction to $\Omega_{T}^{(A, \gamma)}$, with $\bar{\Phi}_{T}$ defined by (2.16).

That $\bar{\Phi}_{T}=0$ when $N_{T}=0$ or $N_{T}=1$ is obvious, and that $\bar{\Phi}_{T} \leq 0$ everywhere follows from the Cauchy-Schwarz inequality. Finally, let us introduce the simplifying notation

$$
a_{m}^{-}=c_{S_{m}-} \phi_{1}^{\prime}\left(c_{S_{m}-}, c_{S_{m}}\right), \quad a_{m}^{+}=c_{S_{m}} \phi_{2}^{\prime}\left(c_{S_{m}-}, c_{S_{m}}\right), \quad a_{m}=\psi\left(X_{S_{m}-}, X_{S_{m}}\right) .
$$

Then (2.16) can be rewritten as

$$
\bar{\Phi}_{T}=2\left(\sum_{m=1}^{N_{T}} a_{m}\left(a_{m}^{-} V_{m}^{-}+a_{m}^{+} V_{m}^{+}\right)\right)^{2}-2\left(\sum_{m=1}^{N_{T}} a_{m}^{2}\right)\left(\sum_{m=1}^{N_{T}}\left(a_{m}^{-} V_{m}^{-}+a_{m}^{+} V_{m}^{+}\right)^{2}\right) .
$$

Since $a_{m} \neq 0$ for all $m$, if $\bar{\Phi}_{T}=0$, we must have

$$
\frac{a_{m}^{-} V_{m}^{-}+a_{m}^{+} V_{m}^{+}}{a_{m}}=\frac{a_{1}^{-} V_{1}^{-}+a_{1}^{+} V_{1}^{+}}{a_{1}}
$$

for all $m=2,3, \ldots, N_{T}$. Since $\left|a_{m}^{-}\right|+\left|a_{m}^{+}\right|>0$ for all $m$, whereas all $V_{m}^{+}$and $V_{m}^{-}$are independent and with a density, conditionally on $\left(a_{p}, a_{p}^{-}, a_{p}^{+}\right)_{p \geq 1}$, this property is almost never true on the set $\left\{N_{T} \geq 2\right\}=\Omega_{T}^{A}$. Therefore, $\bar{\Phi}_{T}<0$ a.s. on $\Omega_{T}^{A}$, and the proof is complete.

Note that, since $\rho<\frac{1}{2},(2.14)$ is always true when $r \leq \frac{4}{3}$. Otherwise, and since the number $r$ is typically unknown, we need to know beforehand an upper bound on it: the closer $r$ is to 2 , the more close to a Brownian motion the compensated sum of jumps is (apart from the 'big' jumps, of course). So, in practice, we should say that although $r$ is unknown, we 'know' (or pretend) that it is smaller than some prescribed value $r_{0}<2$. Then we choose $\rho=\left(2-r_{0}\right) / r_{0}$ if $r_{0}>\frac{4}{3}$ for example.

The limit $\bar{\Phi}_{T}$ in (2.16) depends on the values $X_{S_{m}}, X_{S_{m}-}, c_{S_{m}}$, and $c_{S_{m}-}$, which are not observed. However, we can 'approximate' this limit in the same way as for the consistency above in the following sense. We endow the auxiliary space $\left(\Omega^{\prime}, \mathcal{F}^{\prime}, \mathrm{P}^{\prime}\right)$ with another double sequence $\left(\tilde{V}_{m}^{-}, \tilde{V}_{m}^{+}\right)_{m \geq 1}$ of variables, with the same description as the sequence $\left(V_{m}^{-}, V_{m}^{-}\right)$; if one wants to, one can take the same two double sequences because only their laws matter. 
Theorem 2.2. Assume that the assumptions of Theorem 2.1 hold, and define

$$
\begin{aligned}
\bar{\Phi}_{T}^{n}= & 2\left(\sum_{i=k_{n}+1}^{\left[T / \Delta_{n}\right]-k_{n}} \mathbf{1}_{A}\left(\Delta_{i}^{n} X\right) \psi\left(X_{(i-1) \Delta_{n}}, X_{i \Delta_{n}}\right)\right. \\
& \left.\times\left(\hat{c}_{i-k_{n}-1}^{n} \phi_{1}^{\prime}\left(\hat{c}_{i-k_{n}-1}^{n}, \hat{c}_{i}^{n}\right) \tilde{V}_{i}^{-}+\hat{c}_{i}^{n} \phi_{2}^{\prime}\left(\hat{c}_{i-k_{n}-1}^{n}, \hat{c}_{i}^{n}\right) \tilde{V}_{i}^{+}\right)\right)^{2} \\
& -2\left(\sum_{i=k_{n}+1}^{\left[T / \Delta_{n}\right]-k_{n}} \mathbf{1}_{A}\left(\Delta_{i}^{n} X\right) \psi\left(X_{(i-1) \Delta_{n}}, X_{i \Delta_{n}}\right)^{2}\right) \\
& \times\left(\sum_{i=k_{n}+1}^{\left[T / \Delta_{n}\right]-k_{n}} \mathbf{1}_{A}\left(\Delta_{i}^{n} X\right)\left(\hat{c}_{i-k_{n}-1}^{n} \phi_{1}^{\prime}\left(\hat{c}_{i-k_{n}-1}^{n}, \hat{c}_{i}^{n}\right) \tilde{V}_{i}^{-}+\hat{c}_{i}^{n} \phi_{2}^{\prime}\left(\hat{c}_{i-k_{n}-1}^{n}, \hat{c}_{i}^{n}\right) \tilde{V}_{i}^{+}\right)^{2}\right) .
\end{aligned}
$$

Then the $\bar{\Phi}_{T}^{n}$ are nonpositive variables. Moreover, on the whole space $\Omega$, and not only on $\Omega_{T}^{(A, \gamma)}$,

$$
\bar{\Phi}_{T}^{n} \stackrel{\mathcal{L}-(s)}{\longrightarrow} \Phi_{T}
$$

Proof. The property $\bar{\Phi}_{T}^{n} \leq 0$ again follows from the Cauchy-Schwarz inequality. The rest of the proof is the same as for Theorem 2.1, upon observing that if (2.18) holds, the sequence $\left(\tilde{V}_{i(n, m)}^{-}, \tilde{V}_{i(n, m)}^{+}\right)_{m \geq 1}$ is independent of $\mathcal{F}$ and has the same distribution as the sequence $\left(V_{m}^{-}, V_{m}^{+}\right)_{m \geq 1}$.

\section{Examples}

We shall investigate four model classes for the volatility as listed in the introduction: the Lévy-driven Ornstein-Uhlenbeck model (also called the BNS model in finance) [5], more general CARMA models [7], with the $\operatorname{CARMA}(2,1)$ model as a specific example (cf. [7], and [25]), the COGARCH$(1,1)$ model [19], [21] and its higher-order versions [7], [8], and ECOGARCH models [12]. For readable review papers on continuous-time volatility models, we refer the reader to [20] and [23]. Lévy processes are the natural driving processes in all continuous-time jump models.

In most of the models below the classical Gaussian semimartingale setup for the price process has been extended by a leverage term to allow for the possibility of an upwards jump in the price, whenever there is a jump in the volatility (cf. [5, Equation (8)], [7, Equation (1.2)], and [13, Equation (5.1)]). In all these models all jumps of $X$ and $\sigma^{2}$ are common jumps, since they are caused by the same driving Lévy process $L$.

Example 3.1. (Ornstein-Uhlenbeck or BNS model; cf. [5].) The BNS model suggests a continuous-time semimartingale as the pricing model, and an Ornstein-Uhlenbeck or CAR(1) model driven by a subordinator $L$ as the stochastic volatility, i.e.

$$
X_{t}=X_{0}+\int_{0}^{t} b_{s} \mathrm{~d} s+\int_{0}^{t} \sigma_{s} \mathrm{~d} B_{s}+\rho L_{t}, \quad \sigma_{t}^{2}=\sigma_{0}^{2}+\int_{0}^{t} \mathrm{e}^{-\lambda(t-s)} \mathrm{d} L_{t},
$$

where $\lambda>0$ and $\rho \leq 0$. Here all price jumps are negative and coincide with a positive jump in the volatility. 
Example 3.2. (CARMA $(p, q)$ model; $c f$. [7] and [25].) Motivated to obtain a more flexible autocorrelation function for the stochastic volatility process, Brockwell [7] and Todorov and Tauchen [25] suggested a generalisation of the $\operatorname{CAR}(1)$ model to a general $\operatorname{CARMA}(p, q)$ model. The price model can be the same as in Example 3.1 and the volatility models are, in the CARMA framework, given as

$$
X_{t}=X_{0}+\int_{0}^{t} b_{s} \mathrm{~d} s+\int_{0}^{t} \sigma_{s} \mathrm{~d} B_{s}+\rho L_{t}, \quad \sigma_{t}^{2}=Y_{t},
$$

where $\rho \leq 0, L_{t}$ is a subordinator, and $Y_{t}$ is a CARMA $(p, q)$ process driven by $L_{t}$.

Since volatility models have to be positive, not all CARMA models (even when driven by a subordinator) can be chosen as volatility models. For certain sets of parameters a subordinatordriven CARMA $(2,1)$ model is nonnegative, and has been suggested as a volatility model in [25].

The resulting equation for the squared volatility is

$$
\sigma_{t}^{2}=\sigma_{0}^{2}+\int_{0}^{t} g(t-u) \mathrm{d} L(u)
$$

and the kernel $g(u)$ can be given explicitly. The CARMA $(2,1)$ process has for negative and different roots $\rho_{1}, \rho_{2}$ of $a(\cdot)$ the kernel

$$
g(h)=\frac{1+b_{1} \rho_{1}}{\rho_{1}-\rho_{2}} \mathrm{e}^{\rho_{1} h}+\frac{1+b_{1} \rho_{2}}{\rho_{2}-\rho_{1}} \mathrm{e}^{\rho_{2} h}, \quad h \geq 0 .
$$

It is nonnegative if and only if $0 \leq b_{1} \leq \max \left\{-1 / \rho_{1},-1 / \rho_{2}\right\}$. Hence, for this set of parameters, the CARMA $(2,1)$ model is a possible volatility model, which generalizes the CAR(1) model of Example 3.1 in allowing for more flexible autocorrelation functions. Again, all price jumps are negative and coincide with a positive jump in the volatility.

Example 3.3. (COGARCH $(p, q)$ process; cf. [13] and [19].) The $\operatorname{COGARCH}(1,1)$ model has been suggested as a continuous-time version of the $\operatorname{GARCH}(1,1)$ model by replacing the discrete noise variables by the jumps of a Lévy process $L$. Introducing a possible leverage term results in the following model, and we also include a drift in the price process for completeness:

$$
\begin{aligned}
X_{t} & =X_{0}+\int_{0}^{t} b_{s} \mathrm{~d} s+\int_{0}^{t} \sigma_{s-} \mathrm{d} L_{s}+\rho \sum_{s \leq t, \Delta L_{s}<0} \sigma_{s-}^{2}\left(\Delta L_{s}\right)^{2}, \\
\sigma^{2} t & =\sigma_{0}^{2}+\int_{0}^{t}\left(\beta-\eta \sigma_{s}^{2}\right) \mathrm{d} s+\varphi \sum_{s \leq t} \sigma_{s-}^{2}\left(\Delta L_{s}\right)^{2} .
\end{aligned}
$$

Here $\beta>0, \eta>0, \varphi \geq 0$, and $\rho \geq 0$. Extensions to higher-order COGARCH models have been suggested in [8], but this does not change the relation of the jumps in the price and volatility as given in Proposition 3.1 below. Here the price can have positive and negative jumps, but negative jumps have a higher impact than positive jumps by the leverage term.

Example 3.4. (ECOGARCH processes; cf. [12].) Another model, which involves a CARMA model, but takes care of the necessary nonnegativity of the volatility, models $\log \sigma^{2}$ by a CARMA model, i.e.

$$
X_{t}=X_{0}+\int_{0}^{t} b_{s} \mathrm{~d} s+\int_{0}^{t} \sigma_{s-} \mathrm{d} L_{s}, \quad \sigma_{t}^{2}=\mathrm{e}^{Y_{t}},
$$

where $Y_{t}$ is a CARMA $(q, p-1)$ process with mean $\mu$. This model has a leverage effect inbuilt. 
In COGARCH and ECOGARCH models it is immediate from their definitions that the models allow for common jumps in the price and volatility.

Proposition 3.1. With the definitions given in Examples 3.1-3.4 we obtain the following functional relationships.

- In all the linear models of Examples 3.1 and 3.2, (2.2) holds with $A=\mathbb{R} \backslash\{0\}$ and

$$
\psi_{\mathrm{OU}}(x, y)=\phi_{\mathrm{OU}}(x, y)=y-x
$$

(in these models, the jumps of $X$ are always negative and those of $\sigma^{2}$ positive).

- In all COGARCH models, (2.2) holds with $A=\mathbb{R} \backslash\{0\}$ and

$$
\psi_{\mathrm{COG}}(x, y)=(y-x)^{2}, \quad \phi_{\mathrm{COG}}(x, y)=y-x,
$$

and with $A=(0, \infty)$ and $A=(-\infty, 0)$, with two different values of the constant $\gamma$ on those two sets.

- For the ECOGARCH models, (2.2) holds with

$$
\psi_{\mathrm{ECOG}}(x, y)=y-x, \quad \phi_{\mathrm{ECOG}}(x, y)=\sqrt{x}(\log y-\log x),
$$

and with $A=(0, \infty)$ and $A=(-\infty, 0)$, with two different values of the constant $\gamma$ on those two sets.

\section{Test for a functional relationship}

As already mentioned, we want to test whether the observed outcome lies in the set $\Omega_{T}^{(A, \gamma)}$ of (2.6) for a given pair of functions $(\phi, \psi)$. The 'natural' alternative is then $\Omega_{T}^{A} \backslash \Omega_{T}^{(A, \gamma)}$. This is not the complementary set of $\Omega_{T}^{(A, \gamma)}$, since outside $\Omega_{T}^{A}$ there is 'always' a functional relationship, which is in fact meaningless for the model.

We recall that testing the null hypothesis 'we are in a subset $\Omega_{0}$ ' of $\Omega$ against the alternative 'we are in a subset $\Omega_{1}$ ', with, of course, $\Omega_{0} \cap \Omega_{1}=\varnothing$, amounts to finding a critical (rejection) region $C_{n} \subset \Omega$ at stage $n$. The asymptotic size for this sequence $C_{n}$ of critical regions is the number

$$
\alpha=\sup \left\{\limsup _{n \rightarrow \infty} \mathrm{P}\left(C_{n} \mid A\right): A \in \mathcal{F}, A \subset \Omega_{0}, \mathrm{P}(A)>0\right\} .
$$

Moreover, we say that the sequence $C_{n}$ is consistent for the alternative $\Omega_{1}$ if

$$
\mathrm{P}\left(\left(C_{n}\right)^{\mathrm{c}} \cap \Omega_{1}\right) \rightarrow 0
$$

(where $\left(C_{n}\right)^{\mathrm{c}}$ is the complement of $C_{n}$ in $\Omega$ ). The latter also amounts to saying that $\mathrm{P}\left(C_{n} \mid A\right) \rightarrow$ 1 as $n \rightarrow \infty$ for all $A \subset \Omega_{1}$ having $\mathrm{P}(A)>0$.

The previous limiting results allow us to construct a test with a given asymptotic level $\alpha \in(0,1)$ for the null hypothesis $\Omega_{T}^{(A, \gamma)}$. The procedure is as follows, at any given stage $n$. We single out the following two cases.

(a) We have $\Delta_{i}^{n} X \notin A$ for all $i \leq\left[T / \Delta_{n}\right]$ except perhaps one value of $i$. Then, at stage $n$, it 'looks like' there is 0 or 1 jump of $X$ with size in $A$, and we cannot perform the test at all (asymptotically, as $\Delta_{n} \rightarrow 0$, this cannot occur if we are inside $\Omega_{T}^{A}$ ).

(b) We have $\Delta_{i}^{n} X \in A$ for at least two values of $i$ smaller than $\left[T / \Delta_{n}\right]$. Then we proceed. 
We simulate $M$ copies of the variables $\left(V_{i}^{-}, V_{i}^{+}\right)$and substitute them into (2.22), always with the same observed values of the increments $\Delta_{i}^{n} X$. This gives a sequence $\left(\bar{\Phi}_{T, j}^{n}: j=1, \ldots, M\right)$ of variables which, conditionally on the partially observed path, is i.i.d. with the same (conditional) law as $\bar{\Phi}_{T}^{n}$ in (2.22). In particular, the simulated values $\bar{\Phi}_{T, j}^{n}$ are all distinct (a.s.). We then consider the empirical $\alpha$-quantile

$$
\hat{A}_{n, M}(\alpha)=\bar{\Phi}_{n,([\alpha M])},
$$

where

$$
\bar{\Phi}_{T,(1)}^{n}<\bar{\Phi}_{T,(2)}^{n}<\cdots<\bar{\Phi}_{T,(M)}^{n}
$$

is the increasing reordering of the sequence $\left(\bar{\Phi}_{T, j}\right)_{1 \leq j \leq M}^{n}$. Then $\hat{A}_{n, M}(\alpha)$ converges to the (random, $\mathcal{F}$-measurable) number $A_{n}(\alpha)$ satisfying, as $M \rightarrow \infty$,

$$
\tilde{\mathrm{P}}\left(\bar{\Phi}_{T}^{n}<A_{n}(\alpha) \mid \mathcal{F}\right)=\alpha .
$$

Doing this with a number of simulations $M=M_{n}$, we can take the critical region

$$
C_{n}=\left\{\Phi_{T}^{n}<\frac{\hat{A}_{n, M_{n}}(\alpha)}{k_{n}}\right\} .
$$

Theorem 4.1. Suppose that Assumption (A-r) for some $r \in[0,2),(2.4)$, and (2.5) hold. Then, and as soon as $M_{n} \rightarrow \infty$, the critical regions defined by (4.1) have the asymptotic level $\alpha$ for testing the null hypothesis $\Omega_{T}^{(A, \gamma)}$, and are consistent for the (partial) alternative $\Omega_{T}^{A} \backslash \Omega_{T}^{(A, \gamma)}$.

Proof. First, $A_{n}(\alpha)$ converges in probability to the $\alpha$-quantile $A(\alpha)$ of $\bar{\Phi}$ knowing $\mathcal{F}$, and this is true under the null, and under the alternative as well. So, since, by Theorem 2.1, $k_{n} \Phi_{T}^{n}$ converges stably in law to $\bar{\Phi}_{T}$ under the null and to $-\infty$ under the alternative (because $\Phi_{n} \stackrel{\mathrm{P}}{\rightarrow} \Phi<0$ on $\left.\Omega_{T}^{A} \backslash \Omega_{T}^{(A, \gamma)}\right)$ ), the proof is the same as for Theorem 4.2 of [17] for example.

\section{Conclusion}

A simulation study has shown that the test procedure works reasonably well. However, a real data analysis revealed that such a simple functional relationship cannot be found in the data. For the COGARCH model, the statistical analysis of [18] (cf. Table 2 therein) gave a partially positive answer, where a test for noncorrelation between common jumps in the price and the squared volatility was not rejected even on a $2 \%$ level in the case of the SPDR S\&P 500 ETF (SPY), an exchange traded fund that tracks the S\&P 500 index. The possibility to test for a finite number of factors or regimes $\gamma$ is currently under investigation.

\section{Acknowledgements}

This work was done while J.J. visited the TUM Institute for Advanced Study (TUM-IAS) at the Technische Universität München. He thanks the institute for its hospitality and the Humboldt Association for making this visit possible by a Humboldt Research Award. C.K. thanks the TUM-IAS for financial support.

\section{References}

[1] Aït-Sahalia, Y. (2002). Telling from discrete data whether the underlying continuous-time model is a diffusion. J. Finance 57, 2075-2112.

[2] AïT-Sahalia, Y. ANd JACOD, J. (2007). Volatility estimators for discretely sampled Lévy processes. Ann. Statist. 35, 355-392. 
[3] Aït-Sahalia, Y. and Jacod, J. (2009). Testing for jumps in a discretely observed process. Ann. Statist. 37, 184-222.

[4] Anderson, T. G., Bollerslev, T. and Diebold, F. X. (2003). Some like it smooth, and some like it rough. Tech. Rep., Northwestern University.

[5] Barndorff-Nielsen, O. E. And Shephard, N. (2001). Non-Gaussian Ornstein-Uhlenbeck-based models and some of their uses in financial economics (with discussion). J. R. Statist. Soc. B 63, 167-241.

[6] BarndorfF-Nielsen, O. E. AND ShePhard, N. (2006). Econometrics of testing for jumps in financial economics using bipower variation. J. Financial Econometrics 4, 1-30.

[7] Brockwell, P. J. (2001). Lévy-driven CARMA processes. Ann. Inst. Statist. Math. 53, 113-124.

[8] Brockwell, P. J., ChadraA, E. and Lindner, A. (2006). Continuous-time GARCH processes. Ann. Appl. Prob. 16, 790-826.

[9] Carr, P., Geman, H., Madan, D. And Yor, M. (2003). The fine structure of asset returns: an empirical investigation. J. Business 75, 305-333.

[10] Eberlein, E. ANd Keller U. (1995). Hyperbolic distributions in finance. Bernoulli 1, 281-299.

[11] Fasen, V., Klüppelberg, C. And Lindner, A. (2006). Extremal behavior of stochastic volatility models. In Stochastic Finance, eds A. N. Shiryaev et al., Springer, New York, pp. 107-155.

[12] Haug, S. and Czado, C. (2007). An exponential continuous-time GARCH process. J. Appl. Prob. 44, $960-976$.

[13] Haug, S., Klüppelberg, C., Lindner, A. and Zapp, M. (2007). Method of moment estimation in the COGARCH $(1,1)$ model. Econom. J. 10, 320-341.

[14] Huang, X. and Tauchen, G. (2005). The relative contribution of jumps to total price variance. J. Financial Econometrics 3, 456-499.

[15] Jacod, J. ANd Protter P. (2011). Discretization of Processes. Springer, Berlin.

[16] JACOD, J. AND Shiryaev, A. N. (2003). Limit Theorems for Stochastic Processes, 2nd edn. Springer, Berlin.

[17] Jacod, J. And Todorov, V. (2010). Do price and volatility jump together? Ann. Appl. Prob. 20, 1425-1469.

[18] Jacod, J., KlüPPElberg, C. AND MÜlleR, G. (2012). Testing for non-correlation between price and volatility jumps. Submitted.

[19] KlüPpelberg, C., Lindner, A. And Maller, R. (2004). A continuous-time GARCH process driven by a Lévy process: stationarity and second-order behaviour. J. Appl. Prob. 41, 601-622.

[20] Klüppelberg, C., Lindner, A. And Maller, R. (2006). Continuous time volatility modelling: COGARCH versus Ornstein-Uhlenbeck models. In From Stochastic Calculus to Mathematical Finance, eds Y. Kabanov, R. Lipster and J. Stoyanov, Springer, Berlin, pp. 393-419.

[21] Klüppelberg, C., Maller, R. and Szimayer, A. (2011) The COGARCH: a review, with news on option pricing and statistical inference. In Surveys in Stochastic Processes, eds J. Blath, P. Imkeller and S. Roelly, Eur. Math. Soc., Zürich, pp. 29-58.

[22] LeE, S. S. AND MyKLAND, P. A. (2008). Jumps in financial markets: a new nonparametric test and jump dynamics. Rev. Financial Studies 21, 2535-2563.

[23] Lindner, A. (2009). Continuous time approximations to GARCH and stochastic volatility models. In Handbook of Financial Time Series, eds T. G. Andersen et al., Springer, Berlin, pp. 481-496.

[24] Merton, R. C. (1976). Option pricing when underlying stock returns are discontinuous. J. Financial Econom. 3, 125-144.

[25] Todorov, V. And Tauchen, G. (2006). Simulation methods for Lévy-driven CARMA stochastic volatility models. J. Business Econom. Statist. 24, 450-469. 